\section{Utilização de diagramas causais em epidemiologia: um exemplo de aplicação em situação de confusão}

\author{
Use of causal diagrams in Epidemiology: \\ application to a situation with confounding
}

\author{
Utilización de diagramas causales en epidemiología: \\ un ejemplo de su aplicación en situación \\ de confusión
}

\begin{abstract}
Resumo
Apesar do crescente reconhecimento de seu potencial, os diagramas causais ainda são pouco utilizados na investigação epidemiológica. Uma das possíveis razões é que muitos programas de investigação envolvem temas sobre o qual há certo grau de incerteza sobre os mecanismos dos processos que geram os dados. Neste trabalho, a relação entre estresse ocupacional e obesidade é utilizada como um exemplo de aplicação de diagramas causais em questões relacionadas ao confundimento. São apresentadas etapas da seleção de variáveis para ajuste estatístico e da derivação das implicações estatísticas de um diagrama causal. A principal vantagem dos diagramas causais é tornar explícitas as hipóteses adjacentes ao modelo considerado, permitindo que suas implicações possam ser analisadas criticamente, facilitando, dessa forma, a identificação de possíveis fontes de viés e incerteza nos resultados de um estudo epidemiológico.
\end{abstract}

Causalidade; Métodos Epidemiológicos; Viés (Epidemiologia); Modelos Estatísticos
Taísa Rodrigues Cortes 1

Eduardo Faerstein 1

Claudio José Struchiner 1,2

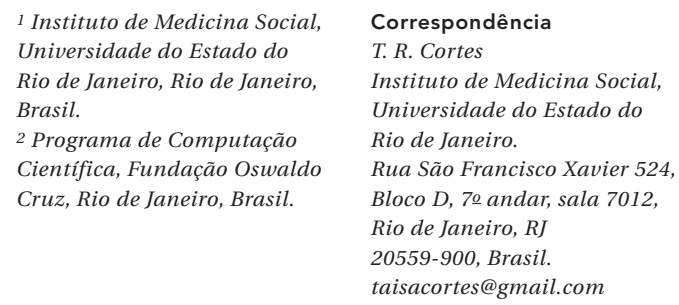




\section{Introdução}

Os diagramas causais têm tido uso crescente como uma técnica unificada para lidar com uma série de questões na pesquisa epidemiológica 1. Esses modelos gráficos proporcionaram novas formalizações para alguns conceitos epidemiológicos importantes, como a noção de confundimento ${ }^{2}$, viés de seleção 3 e viés de informação 4 . Além disso, têm sido utilizados como ferramentas auxiliares na análise de dados faltantes 5 e na análise de mediação 6 .

Apesar do crescente reconhecimento de seu potencial, os diagramas causais ainda são pouco utilizados na investigação epidemiológica. Uma das possíveis razões é que muitos programas de investigação envolvem temas sobre o qual há certo grau de incerteza sobre os mecanismos dos processos que geram os dados. Neste artigo, a relação entre estresse no trabalho e obesidade é utilizada como um exemplo de aplicação dos diagramas causais em questões relacionadas ao confundimento; seu objetivo não é validar ou contestar hipóteses específicas sobre as relações entre essas variáveis, mas sim utilizar os diagramas causais para discutir implicações de diferentes conjuntos de hipóteses. São apresentadas etapas da seleção de variáveis para ajuste estatístico e da derivação das implicações estatísticas de um diagrama causal. Espera-se fornecer subsídios e colaborar para divulgação da técnica ainda pouco explorada por pesquisadores brasileiros.

\section{A teoria dos diagramas causais}

Componentes da teoria dos diagramas causais foram desenvolvidos em diferentes momentos da história de diferentes áreas de conhecimento 7,8 . No entanto, a popularização desses modelos gráficos na análise causal teve início, especialmente, com trabalhos no campo da Inteligência Artificial, desenvolvidos por Pearl 9,10, e na Filosofia ${ }^{11}$.

Os diagramas causais são gráficos acíclicos direcionados que codificam hipóteses qualitativas sobre os processos causais que geram os dados. A seguir são apresentados a terminologia dos gráficos acíclicos direcionados, a representação das hipóteses causais no gráfico e os pressupostos que permitem avaliar como essas hipóteses causais se relacionam com os dados observados.

\section{Gráfico acíclico direcionado}

Um gráfico acíclico direcionado (Directed Acyclic Graph - DAG) é composto por vértices que re- presentam as variáveis aleatórias e por arestas que denotam uma relação entre um par de variáveis. Um gráfico é direcionado quando todas as arestas são representadas por uma seta única e é acíclico quando nenhuma ligação entre variáveis forma um circuito fechado.

Em um DAG, cada seta possui uma origem (vértice inicial) e um destino (vértice final). A variável origem é denominada de variável pai e a variável de destino é chamada de filha. Um caminho é uma sequência de arestas adjacentes, ou seja, uma sequência de setas em qualquer direção. Se cada aresta em um caminho representa uma seta que aponta do primeiro para o segundo vértice, temos um caminho direto. Variáveis que interceptam o caminho direto entre dois vértices são chamadas de variáveis mediadoras. Uma variável é chamada de colisor quando, em um mesmo caminho, duas setas apontam para essa variável.

Se existe um caminho entre dois vértices representados no gráfico, então os vértices estão conectados; caso contrário, estão desconectados. Se há um caminho direto de $\mathrm{A}$ até $\mathrm{B}$, dizemos que A é um ancestral de B, e B é um descendente de A.

\section{DAG e causalidade}

A utilização de um DAG na modelagem causal reforça a noção de que a causalidade implica direcionalidade de influência. Vale dizer, que a relação causal é uma relação assimétrica em que a causa influencia o desfecho e não o oposto. Em um DAG, a presença de uma seta entre duas variáveis X eY representa a possibilidade de uma relação causal direta entre essas variáveis. Vale ressaltar que qualquer mecanismo causal pode ser decomposto em mecanismos mais detalhados e, assim, uma causa será "direta" ou "indireta" com relação às outras variáveis que compõem um modelo causal. Por outro lado, as principais hipóteses causais estão codificadas pelas ligações (setas) ausentes no diagrama. A ausência de uma seta entre duas variáveis X e Y representa a hipótese mais definitiva de efeito causal direto nulo.

Em situações em que a causação mútua (ciclos causais) entre eventos é possível, a direcionalidade causal pode ser restaurada se a sequência temporal dos eventos for definida. Por exemplo, o ciclo causal entre duas variáveis $\mathrm{X} \mathrm{eY}$, representado na Figura 1a, pode ser decomposto como uma cadeia de causações sucessivas entre instâncias dessas variáveis no tempo (Figura 1b) e essas representações podem ser expandidas para diferentes conjuntos de variáveis e diferentes períodos de tempo. Porém, dependendo do sistema causal em estudo e dos objetivos da mo- 
Figura 1

(1a) Gráfico representando o ciclo causal entre duas variáveis $X$ e Y; (1b) Gráfico acíclico direcionado representando as relações entre as variáveis $X(t)$ e $Y(t)$ no tempo $t$.

1a)

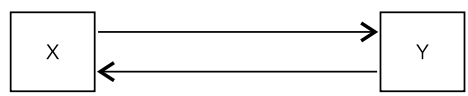

1b)

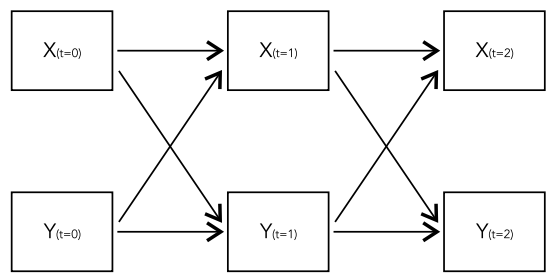

delagem, diferentes estratégias de representação de ciclos causais podem ser adotadas 11,12,13,14.

O significado dessas noções causais codificadas em um diagrama é dado pelo conceito de intervenção. Uma intervenção é definida em Pearl 10 como uma "cirurgia atômica" que remove inteiramente os mecanismos que determinam uma variável, enquanto preserva as demais relações no modelo. Isso é, uma intervenção imposta a uma variável $X$ é aquela que interrompe completamente o relacionamento entre $\mathrm{X}$ e seus pais (causas diretas) e define um novo valor constante $(X=x)$. Além disso, essa intervenção é "cirúrgica", pois não altera as demais relações causais no diagrama. Na linguagem gráfica, esse tipo de intervenção é chamado de intervenção ideal e é denotado por do $(\mathrm{X}=\mathrm{x}) 10$.

A noção expressa por "do $(X=x)$ ", análoga à noção de "experimento ideal", tem a função de um operador matemático que permite formalizar e derivar implicações de diferentes noções causais. Em geral, os parâmetros causais envolvem a comparação entre diferentes distribuições de probabilidade para o desfecho sob diferentes intervenções em X. Por exemplo, para dois conjuntos disjuntos de variáveis $\mathrm{X}$ eY, o efeito causal de $\mathrm{X}$ em Y, denotado por $\operatorname{Pr}(\mathrm{Y} \mid$ do $(\mathrm{X}=\mathrm{x}))$, é dado pela distribuição de probabilidade de $\mathrm{Y}$ quando uma intervenção do tipo $\mathrm{X}=\mathrm{x}$ é aplicada sobre a população.
Desse modo, a interpretação causal de um DAG refere-se à capacidade de a estrutura gráfica descrever não somente como o modelo se comporta em condições normais (ausência de intervenção), mas também como o modelo reage a certas intervenções impostas às variáveis no diagrama.

DAG, causalidade e probabilidade: a condição causal de Markov

Para que um diagrama tenha alguma finalidade na epidemiologia, são necessários pressupostos que conectem a estrutura causal, representada pelo DAG, aos dados obtidos em um estudo epidemiológico 15. Essa relação entre causalidade e probabilidades é dada pela condição causal de Markov 10,11.

Seja G um DAG que representa as relações causais entre um conjunto de variáveis $\mathrm{V}$ e $\mathrm{P}$ uma distribuição de probabilidades para as variáveis em V, G e P satisfazem a condição causal de Markov se cada variável em V é independente de seus não descendentes (i.e., não efeitos em $\mathrm{G}$ ) dado o conjunto de seus pais (i.e., causas diretas em G).

Assim, a condição casual de Markov impõe restrições (na forma de relações de independência condicional) sobre a distribuição de probabilidades que pode ser considerada compatível com a estrutura gráfica. Ressalta-se que a capacidade de um DAG e uma distribuição de probabilidades (P) de satisfazerem a condição casual de Markov depende de um conjunto de propriedades compartilhadas por ambos. A seguir são apresentadas as noções implícitas na condição casual de Markov:

(i) a condição casual de Markov é uma generalização do princípio da causa comum descrito por Reichenbach ${ }^{16}$. Tal princípio sugere que, se duas variáveis são probabilisticamente dependentes, então uma é a causa da outra ou essas duas variáveis compartilham uma causa em comum. Em outras palavras, ignorando a variação amostral, não há correlação entre variáveis sem causalidade;

(ii) a condição casual de Markov implica a noção de "localidade" da relação causal. De forma geral, essa noção sugere que dado o conhecimento das causas diretas (mais proximais) de uma variável, as suas causas indiretas (distais) se tornam irrelevantes para se determinar a sua probabilidade. Isso é, ignorando os seus efeitos, toda a informação probabilística relevante sobre uma variável pode ser obtida pelo conjunto contendo todas as suas causas diretas ${ }^{11}$. Contudo, na prática, dificilmente se tem acesso a todas as causas que afetam diretamente uma determinada variável. 
Em vez disso, sempre que uma "causa direta" é representada em um modelo, um número incontável de causas intermediárias é omitida 17. E à medida que essas abstrações são feitas, precisase decidir em que fase as propriedades úteis para a causalidade são perdidas 10 . Desse modo, a condição casual de Markov serve como um guia para avaliar quando o conjunto de pais de uma variável é considerado completo porque inclui todas as suas causas diretas relevantes;

(iii) um conjunto de variáveis é considerado causalmente completo se todas as causas comuns (mensuradas ou não) entre duas ou mais variáveis (no gráfico) estão representadas no DAG. Ou seja, se pode-se assumir que as variáveis (exógenas) omitidas no gráfico são mutuamente independentes, então é possível assegurar que as demais relações causais não codificadas no DAG não influenciam a distribuição de probabilidades para as variáveis no DAG. Desse modo, o modelo causal (o DAG e o conjunto de distribuição de probabilidades) pode ser considerado completo no sentido de ser capaz de explicar todas as associações entre variáveis causalmente independentes (não conectadas por uma seta) por meio de suas causas comuns. Ou seja, que a estrutura gráfica representa todos os determinantes causais das associações estatísticas entre as variáveis no gráfico. Por outro lado, se duas variáveis (X eY) não estão conectadas por uma seta, espera-se que essas variáveis sejam probabilisticamente independentes, dadas as suas causas comuns. Se X e Y estão associadas na população, postula-se que há uma relação causal direta (não representada) entre essas variáveis, ou há uma causa comum oculta. Vale dizer, que a estrutura causal considerada é incompleta;

(iv) a condição casual de Markov é definida não somente em relação a um conjunto de variáveis (causalmente completo), mas também à distribuição populacional. As relações probabilísticas ditadas pela condição casual de Markov podem ou não ser satisfeitas na distribuição amostral, especialmente na presença de viés de seleção. Outra situação que impõe restrições à condição casual de Markov é a presença de indivíduos com diferentes estruturas causais para as variáveis do modelo. Nesses casos, a distribuição de probabilidade P será uma mistura de distintas distribuições resultantes de diferentes estruturas causais 11 . Por exemplo, em geral, a condição casual de Markov não estará satisfeita quando há interferência (ou contágio) entre unidades, isso é, quando os valores de uma variável para uma unidade influenciam (os valores das variáveis de) outras unidades na população. Como ocorre, por exemplo, quando o desfecho é uma doença infecciosa, e o status da exposição para um indivíduo pode influenciar o desfecho dos demais indivíduos na população 18 .

Outra situação que impõe dificuldades é a presença de ciclos causais entre variáveis. Uma das noções implícitas na condição casual de Markov é que o comportamento dinâmico do sistema pode ser decomposto em uma cadeia de causações sucessivas entre as variáveis no tempo. Muitos fenômenos de interesse na epidemiologia consistem em múltiplos mecanismos interagindo entre si, em diferentes níveis e ao longo do tempo. Considerando que a especificação desses modelos é substancialmente mais exigente, em alguns casos, poderá não ser possível representar a dinâmica dessas relações de causa e efeito por meio dos DAG. Outras técnicas baseadas em simulação computacional e modelagem de sistemas oferecem alternativas para o estudo e representação desses fenômenos 19 .

\section{Resultados: um exemplo de aplicação de diagramas causais em epidemiologia}

A seguir são apresentados DAG hipotéticos para exemplificar as etapas da derivação das implicações estatísticas de um diagrama causal e da seleção de variáveis para controle de confundimento.

Como simplificação, neste exemplo, o constructo estresse no trabalho será considerado como uma exposição dicotômica e representado graficamente por um único vértice. No entanto, em alguns casos, as variáveis mensuradas no estudo não corresponderão às variáveis hipotéticas em uma cadeia causal de interesse. Operacionalmente, pode-se escolher indicadores para representar uma variável de interesse que não seja empiricamente observável ou que não tenha sido mensurada. Nesses casos, deve-se incluir no gráfico a variável latente e seus "substitutos".

Além disso, assume-se aqui a ausência de erros de mensuração da exposição, desfecho e demais covariáveis. Entretanto, essa hipótese é pouco plausível em estudos epidemiológicos. Desse modo, diagramas causais realistas devem incluir vértices adicionais para cada variável mensurada com erro 4 . Em muitos casos também será necessário incluir um vértice no gráfico para representar o processo de selecão dos indivíduos (incluindo os procedimentos de seleção no estudo, perdas de seguimento, não resposta etc.) e indicar as variáveis que explícita ou implicitamente afetam a seleção 3,20 .

Em muitos casos será indicado construir mais de um gráfico acíclico direcionado para revelar as principais incertezas sobre as relações entre as variáveis, incluindo a presença ou ausência de setas e a direção das relações 21 . 
Na Figura 2a, assume-se, por exemplo, que o efeito do sexo na exposição ao estresse no trabalho é mediado por fatores socioeconômicos e ocupacionais, como escolaridade, renda e tipo de ocupação. Essas noções estão codificadas no gráfico por meio de diferentes caminhos (por exemplo: o caminho entre sexo $\rightarrow$ escolaridade $\rightarrow$ renda $\rightarrow$ estresse no trabalho).

Na Figura 2b, foi incluída uma seta entre cor da pele/discriminação racial e o estresse no trabalho para representar a hipótese causal de que a cor/raça tem um efeito sobre a exposição ao estresse ocupacional. Essa relação putativa pode ser derivada do aumento dos níveis de estresse, por exemplo, por meio de processos envolvendo discriminação racial no ambiente de trabalho. Note-se aqui que alguns autores apontam limitações acerca da variável "cor da pele" como marcadora de experiências de discriminação 22 . Logo, a escolha inadequada de variáveis ou indicadores para a representação de constructos (como discriminação racial) tem implicações para a validade do modelo considerado.

Também é hipotético que certos traços de personalidade podem influenciar escolhas profissionais, como tipo de ocupação, e comportamentos de saúde associados à obesidade, como a prática de atividade física de lazer e alimentação. A nova seta ligando escolaridade e estresse no trabalho representa a hipótese causal de que a escolaridade tem um efeito sobre a exposição que não é mediada pelas demais variáveis no gráfico.

Ressalta-se que esses diagramas causais não são modelos inteiramente adequados para o tema de pesquisa. As simplificações adotadas visaram facilitar o entendimento da aplicação dos critérios gráficos. No entanto, há ferramentas computacionais disponíveis 23,24 , como o DAGitty (http://www.dagitty.net), que facilitam a aplicação desses critérios gráficos mesmo em modelos mais complexos.

\section{Implicações estatísticas de um diagrama causal: o critério de $d$-separação}

Na seção anterior, foi apresentada a principal condição (CCM) que permite avaliar como uma estrutura causal se relaciona com os dados observados. No entanto, uma maneira mais conveniente de verificar essa compatibilidade é listar as relações de independência condicional implícitas no DAG, por meio de um critério gráfico chamado de $d$-separação ${ }^{10}$.

A ideia principal do conceito de $d$-separação é verificar se o "fluxo de dependência estatística" está ativo ao longo dos caminhos que ligam as variáveis 17. Duas variáveis X e Y podem estar ligadas por muitos caminhos em um gráfico. $\mathrm{X} \mathrm{e}$ Y são $d$-conectadas se houver qualquer caminho aberto entre essas variáveis. Então, X e Y são $d$ separadas se todos os caminhos que as conectam estão bloqueados. Um caminho é dito bloqueado por um conjunto de vértices $\mathrm{Z}$ :

1) se o caminho contém uma conexão em cadeia $(\mathrm{X} \rightarrow \mathrm{b} \rightarrow \mathrm{Y}$ ) ou uma conexão divergente $(\mathrm{X} \leftarrow \mathrm{b}$ $\rightarrow \mathrm{Y}$ ), no qual o vértice central (b) está contido em Z, ou

2) se o caminho contém uma conexão convergente $(\mathrm{X} \rightarrow \mathrm{c} \leftarrow \mathrm{Y}$ ) no qual nem o vértice central (c) e nenhum de seus descendentes estão em $\mathrm{Z}$.

Considere as relações entre três variáveis, $X$, Y e Z, representadas a seguir. Aplicando o critério de $d$-separação pode-se concluir se a variável X é $d$-separada de $\mathrm{Y}$, dado $\mathrm{Z}$.

$$
\begin{aligned}
& \mathrm{X} \rightarrow \mathrm{Z} \rightarrow \mathrm{Y} \\
& \mathrm{X} \leftarrow \mathrm{Z} \rightarrow \mathrm{Y} \\
& \mathrm{X} \rightarrow \mathrm{Z} \leftarrow \mathrm{Y}
\end{aligned}
$$

Nos dois primeiros casos, $\mathrm{X}$ e Y são $d$-separadas por $\mathrm{Z}$, pois o caminho entre $\mathrm{X}$ e $\mathrm{Y}$ é bloqueado quando se condiciona pela variável intermediária nesse caminho (em 1) ou pela causa comum (em 2). Em outras palavras, uma associação marginal entre duas variáveis pode refletir duas estruturas causais distintas - ou há uma relação causal entre essas variáveis ou essas duas variáveis compartilham uma causa comum. Todavia, o fluxo de dependência estatística entre essas variáveis é bloqueado quando se condiciona pela variável intermediária ou pela causa comum Z.

Por outro lado, o caminho representado (em 3) converge em $Z$, ou seja, $Z$ é um vértice colisor no caminho entre X e Y, e, portanto, esse caminho é bloqueado. Assim, X e Y são marginalmente independentes, já que o único caminho entre eles é bloqueado por um vértice colisor. Porém, quando se condiciona pelo colisor $\mathrm{Z}$ (ou por seu descendente), induz-se uma associação entre as variáveis anteriores das quais ele é dependente (efeito). Portanto, X e Y são $d$-conectadas por Z. Ou seja, uma associação entre duas variáveis não pode ser explicada por um evento no futuro (o seu efeito), mas quando se condiciona por um efeito comum de duas causas independentes leva essas causas a se tornarem probabilisticamente associadas, pois se se possui informação sobre uma das causas, a outra se torna mais ou menos provável, dado que o efeito já ocorreu 10.

Como demonstrado em Pearl 10, se duas variáveis X e Y são $d$-separadas por $\mathrm{Z}$ em um DAG $\mathrm{G}$, isso implica que X e Y são condicionalmente independentes dado $\mathrm{Z}$ em toda distribuição de probabilidades compatível com G. Por outro lado, se X e Y são $d$-conectadas por Z em um DAG G, então X e Y são condicionalmente dependen- 
Figura 2

(2a) Gráfico acíclico direcionado representando hipóteses sobre as relações entre exposição, desfecho e demais covariáveis (2b) Gráfico acíclico direcionado representando hipóteses alternativas.

2a)

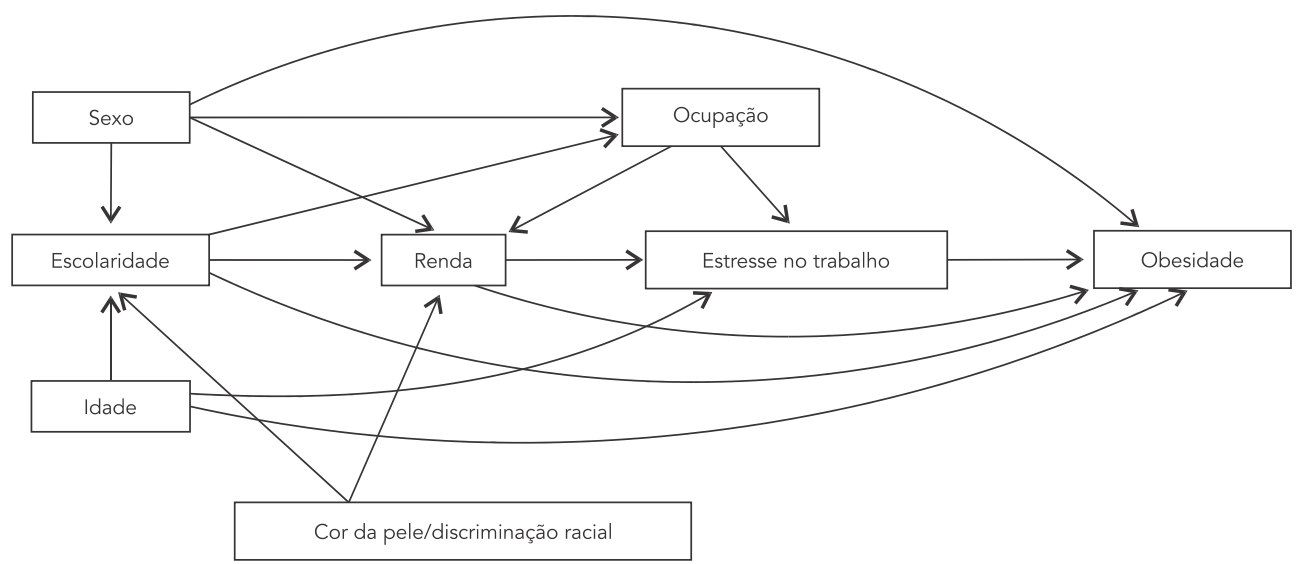

2b)

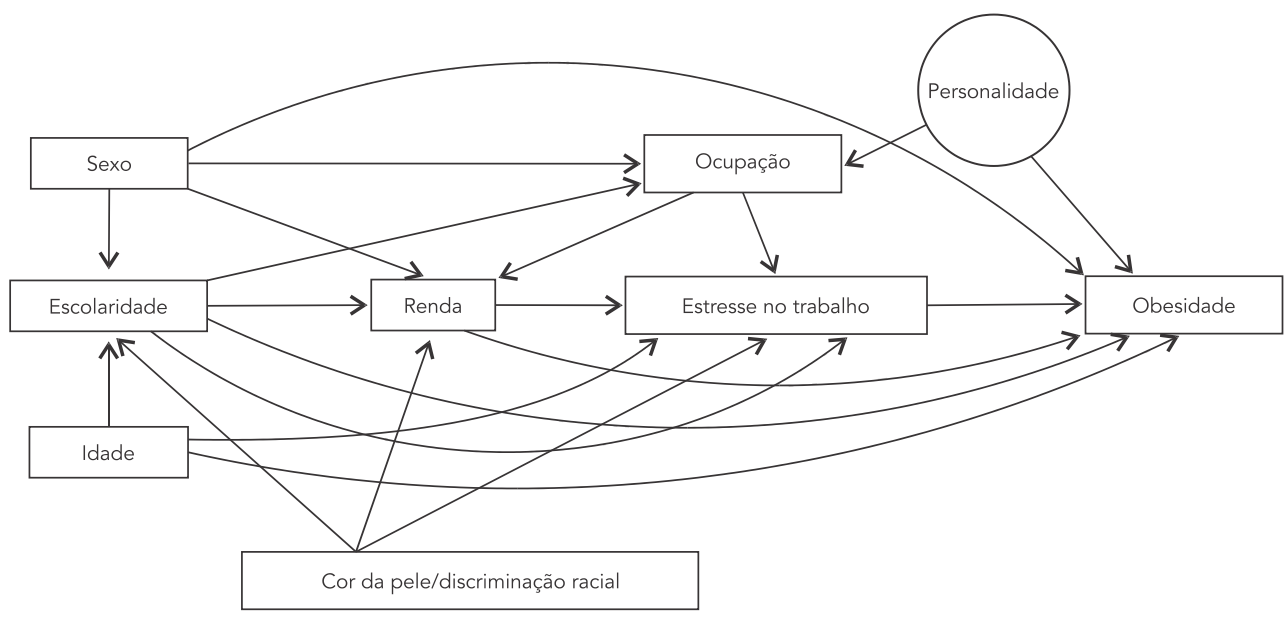

Nota: círculo indica que a variável não foi mensurada.

tes dado $\mathrm{Z}$ em, pelo menos, uma distribuição de probabilidades compatível com G.

A aplicação do critério de $d$-separação pode se tornar uma tarefa difícil quando a estrutura gráfica contém um grande número de vértices e ligações (setas). Nesses casos, é recomendado a utilização de softwares específicos, como o DAGitty, que permitem verificar as implicações estatísticas do modelo 23.

A Figura 3 apresenta as relações de independência condicional definidas pela estrutura do diagrama causal da Figura 2a, por meio dos critérios de d-separação. Em seguida, essas relações estatísticas podem ser avaliadas pelo uso de alguma classe de teste de independência condicional 11.

Se as implicações testáveis não são validadas pelos dados, então o DAG pode não ser uma representação acurada dos processos causais que geram os dados. Uma vez que a estrutura causal "correta" não pode ser inferida por meio de informações puramente probabilísticas, o pes- 


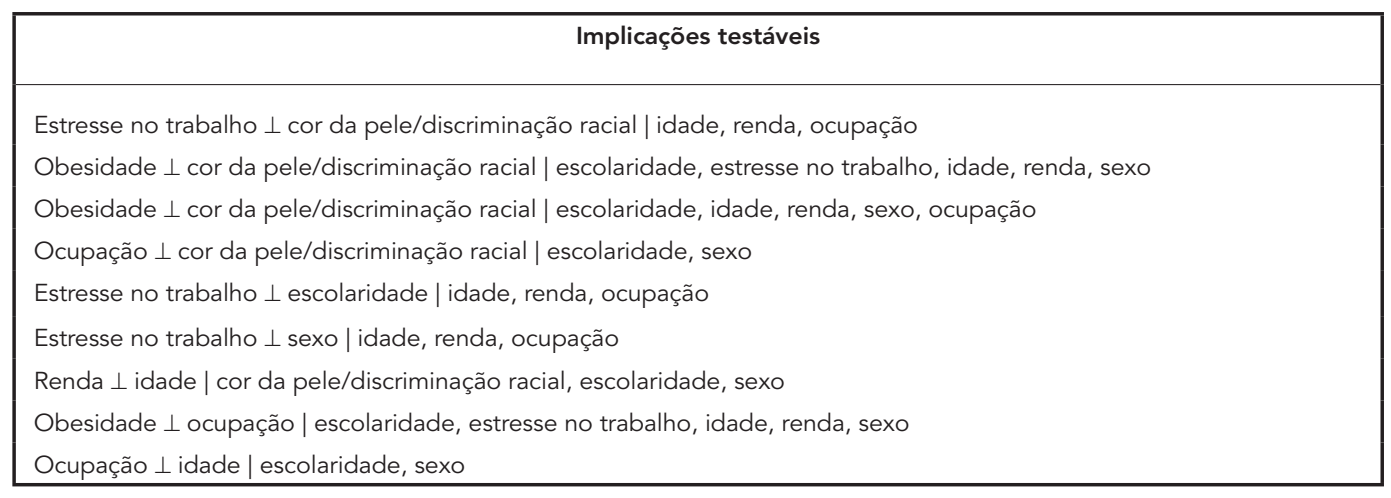

Nota: o símbolo " $\perp$ " indica independência estatística.

quisador deve avaliar cuidadosamente as estratégias de modificação do modelo. Ou seja, além das questões relacionadas aos testes de hipóteses, em especial a possibilidade de erros do tipo I e tipo II, deve-se considerar que um mesmo conjunto de relações de independência condicional é consistente com múltiplos gráficos.

Como descrito em Greenland 20, para cada par de vértices (X e Y) representado no gráfico, assume-se um conjunto de hipóteses nulas que inclui: (i) ausência de causas em comuns entre X e Y não representadas no DAG; (ii) ausência de associação induzida entre X e Y devido ao condicionamento por um efeito em comum não representado no DAG; e (iii) se X eY não são adjacentes (nem $\mathrm{X} \rightarrow \mathrm{Y}$ ou $\mathrm{X} \leftarrow \mathrm{Y}$ ), então não há efeito causal direto entre essas variáveis.

Nenhum desses pressupostos pode ser testado isoladamente por meio dos dados. Desse modo, a ausência de suporte empírico para essas hipóteses representadas em um diagrama implica, muitas vezes, a necessidade de "relaxamento" dos pressupostos causais. Assim, muitos temas de investigação podem exigir diagramas causais complexos, incluindo um grande número de ligações (setas) e vértices para representar variáveis latentes, erros de mensuração e seleção nas análises ${ }^{20}$. Como consequência, a avaliação prévia desses modelos (e a identificação dos parâmetros causais) pode se tornar difícil.
Seleção de variáveis para ajuste estatístico: o critério da porta de trás

Pearl 10 sugere a notação $\operatorname{Pr}(\mathrm{Y} \mid d o(\mathrm{X}=\mathrm{x}))$ e $\operatorname{Pr}(\mathrm{Y} \mid$ $\mathrm{X}=\mathrm{x}$ ) para distinguir entre a distribuição de probabilidade condicional dada uma intervenção em X e dada a observação passiva de X, respectivamente. O primeiro reflete como a distribuição de $\mathrm{Y}$ deve ser modificada, dada a informação de que $\mathrm{X}$ tenha sido manipulado, por alguma intervenção externa, a assumir o valor “x”, enquanto a segunda reflete como a distribuição de Y deve ser modificada, dado que se verifica que $\mathrm{X}=\mathrm{x}$.

Em geral, não se espera que uma intervenção no fator de exposição produza o mesmo efeito que a observação da exposição. Uma das razões para essa distinção refere-se à presença de caminhos não causais que ligam a exposição (E) e o desfecho (D). Em um diagrama, os caminhos diretos entre E e D representam a associação causal entre essas variáveis, enquanto os caminhos não diretos entre essas variáveis podem representar uma fonte de associação espúria. Esses caminhos não causais são chamados de caminhos pela porta de trás (i.e., caminhos que começam com uma causa direta de E - seta pela porta de trás - e terminam em D). O fluxo de dependência estatística entre esses caminhos estará ativo se, e somente se, esses caminhos contêm um ancestral (causa) comum de E e D 2,10. Ou seja, há confundimento quando a exposição e o desfecho compartilham uma ou mais causas em comum. 
Para o controle de confundimento é necessário selecionar um conjunto de covariáveis que permita bloquear todos os caminhos pela porta de trás, enquanto mantém desbloqueados todos os caminhos causais que ligam a exposição e o desfecho.

As principais estratégias tradicionais de seleção dessas covariáveis envolvem combinações de critérios teóricos e estatísticos, como os critérios tradicionais para definição de confundidores 25 , abordagens baseadas em procedimentos automáticos de seleção de variáveis, como as técnicas do tipo stepwise e outros critérios que envolvem a noção de não colapsibilidade de uma medida de efeito 25 .

Alguns autores têm demonstrado que todas as três estratégias podem levar a conclusões inadequadas, devido à omissão de importantes fatores de confusão ou devido ao ajuste impróprio para não confundidores 3,15,26. Diferentes tipos de variáveis, como variáveis intervenientes e colisores, podem comportar-se estatisticamente como fatores de confusão, ainda que, de fato, não o sejam. Por tal razão, a compreensão da estrutura causal é um passo essencial quando pretendemos determinar se a inclusão de uma determinada covariável no conjunto para ajuste é passível de reduzir ou aumentar o viés na estimativa de efeito 27 .

Neste trabalho, é considerada apenas a avaliação do efeito total de uma exposição e um desfecho. No entanto, esses critérios podem ser generalizados para considerar mais de uma exposição e/ou desfecho. Análises de efeitos diretos e indiretos necessitam de regras adicionais descritas em Pearl 6.

Como descrito em Pearl 10, um conjunto de variáveis $\mathrm{S}$ satisfaz o critério da porta de trás com relação a uma exposição (E) e um desfecho (D) em um DAG se: (i) $\mathrm{S}$ não contém nenhum descendente de E; e (ii) as variáveis em S bloqueiam todos os caminhos entre E e D que contêm uma seta apontando para E. Essas noções podem ser identificadas por meio do seguinte algoritmo gráfico 2 :

a) Remova todas as setas que se originam da exposição. Em primeiro lugar, o conjunto S não deve conter nenhum descendente da exposição, isto porque, ao condicionar por algum efeito da exposição que seja interveniente na relação entre a exposição (E) e desfecho (D) será bloqueado algum dos caminhos causais que ligam E a D. Além disso, ao condicionar por efeitos da exposição, pode-se desbloquear ou criar caminhos que não fazem parte do efeito de $\mathrm{E}$ em $\mathrm{D}$, podendo, portanto, introduzir viés. Por exemplo, se uma covariável em S é um efeito comum da exposição e do desfecho, a análise condicionada por S geralmente resultará em uma associação entre essas duas variáveis. Essa associação entre E e D induzida por S é considerada como viés por conta do condicionamento por um colisor (collider stratification bias) 28;

b) Em seguida, junte com uma linha pontilhada quaisquer variáveis que apresentam uma filha em comum que esteja contida em $S$, ou que tenha um descendente em S. Ou seja, a ligação entre duas variáveis por meio da linha pontilhada representa as associações induzidas em razão do ajuste por S. Essas associações induzidas representam novos caminhos que ligam a exposição ao desfecho e, portanto, devem ser incluídos no novo gráfico;

c) No novo gráfico, verifique se existe um caminho aberto de $\mathrm{E}$ para $\mathrm{D}$, que não passa através de um elemento de S. Será considerado como um conjunto suficiente se essas variáveis contidas em $\mathrm{S}$ bloqueiam todos os caminhos (não causais) que ligam a exposição ao desfecho. Para isso, todos os caminhos abertos (caminhos que não contêm colisores) que ligam a exposição ao desfecho devem ser interceptados por uma variável que esteja contida em S.

Considerando-se os diagramas causais na Figura 2, pode-se testar, por exemplo, se as variáveis idade, sexo e renda são suficientes para controle de confundimento. Nas Figuras 4, 5 e 6 são apresentados novos gráficos para cada aplicação do critério da porta de trás.

Na Figura 4, verifica-se que o ajuste por essas variáveis é insuficiente para o controle de confundimento, pois alguns dos caminhos pela porta de trás que ligam a exposição ao desfecho permaneceriam desbloqueados. Nota-se que alguns dos caminhos bloqueados (por exemplo, estresse no trabalho $\leftarrow$ ocupação $\rightarrow$ renda $\leftarrow$ cor da pele/discriminação racial $\rightarrow$ escolaridade $\rightarrow$ obesidade) se tornam ativos por causa do ajuste por um vértice colisor (renda). Como todos esses caminhos passam pelos vértices escolaridade e ocupação, então a inclusão de apenas uma dessas covariáveis no conjunto para ajuste será suficiente para o controle de confundimento. Além disso, se a cor da pele/discriminação racial está relacionada com o estresse no trabalho apenas através de seus efeitos sobre a renda e escolaridade, então o ajuste para esta covariável será desnecessário.

Ou seja, mesmo que um conjunto de variáveis seja considerado suficiente para o controle de confundimento, em algumas situações pode ser desnecessário controlar por todas as variáveis selecionadas ${ }^{2}$. Dessa forma, pode-se verificar se existe um conjunto com menor número de variáveis e que também seja suficiente para o controle de confundimento. 
Gráfico representando a aplicação do critério da porta de trás no diagrama da Figura 2a. O conjunto para ajuste contém as variáveis idade, sexo e renda.

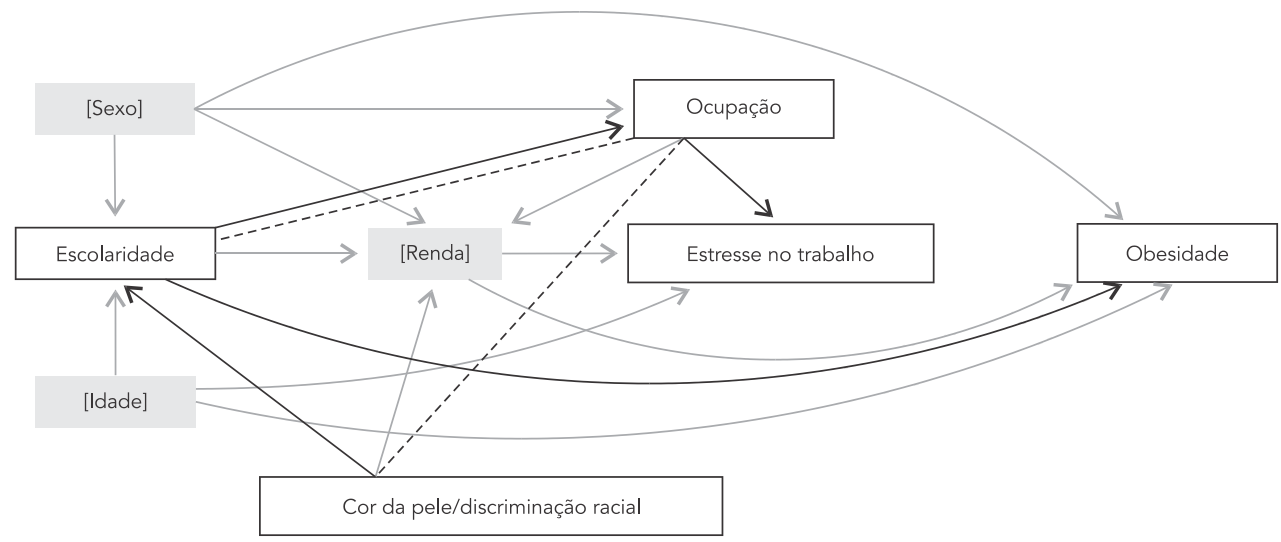

Nota: setas em negrito, destacam-se os caminhos pela porta de trás desbloqueados.

Um conjunto mínimo suficiente é o menor conjunto de variáveis capaz de bloquear todos os caminhos pela porta de trás que ligam a exposição ao desfecho ${ }^{10}$. Uma forma de determinar se existe tal conjunto mínimo e suficiente é excluir algumas variáveis do conjunto candidato para o ajuste e aplicar novamente os passos anteriores.

No diagrama da Figura 2a, com a aplicação do critério da porta de trás, são obtidos quatro conjuntos suficientes para controle de confundimento $S_{1}=$ idade, sexo, renda, escolaridade, ocupação e cor da peleldiscriminação racial; $S_{2}$ = idade, renda, escolaridade, ocupação e cor da pele/discriminação racial; $S_{3}=$ idade, sexo, renda, escolaridade e cor da peleldiscriminação racial; $S_{4}=$ idade, renda, ocupação e cor da pele/discriminação racial e dois conjuntos mínimos e suficientes, $S m_{1}=$ idade, renda e ocupação e $S m_{2}=$ idade, sexo, renda e escolaridade. constata-se que um conjunto contendo todas as causas diretas da exposição será considerado suficiente, mas em alguns casos não será um conjunto mínimo.

Considerando as hipóteses alternativas representadas no diagrama da Figura $2 b$, pode-se testar se esses conjuntos mínimos $\left(\mathrm{Sm}_{1}\right.$ e $\left.\mathrm{Sm}_{2}\right)$ continuam sendo suficientes para controle de confundimento.

Como demonstrado na Figura 5, se a escolaridade e a cor da pele/discriminação racial estão relacionadas com o estresse no trabalho por meio de outros caminhos que não incluem a renda, então as variáveis em $\mathrm{Sm}_{1}$ não são mais suficien- tes para bloquear todos os caminhos pela porta de trás que ligam a exposição ao desfecho. Alguns desses caminhos incluem a variável escolaridade (por exemplo, estresse no trabalho $\leftarrow$ escolaridade $\rightarrow$ personalidade $\rightarrow$ obesidade), os demais caminhos são interceptados pelo vértice cor da pele/discriminação racial (por exemplo, estresse no trabalho $\leftarrow$ cor da pele/discriminação racial $\rightarrow$ personalidade $\rightarrow$ obesidade). Assim, a inclusão dessas duas covariáveis no conjunto para ajuste será suficiente para controle de confundimento.

Da mesma maneira, o ajuste para as variáveis em $\mathrm{Sm}_{2}$ não é mais suficiente para controle de confusão (Figura 6), pois os caminhos que ligam o estresse no trabalho à obesidade, por meio de uma causa comum não observada (personalidade), permanecem desbloqueados. Como esses caminhos passam pelo vértice ocupação, então é necessário incluir essa covariável no conjunto para ajuste. Portanto, se a estrutura causal representada na Figura 2b está correta, então os conjuntos (mínimos), $\mathrm{Sm}_{3}=$ i idade, renda, ocupação, escolaridade e cor da pele/discriminação racial e $\mathrm{Sm}_{4}=$ idade, sexo, renda, escolaridade e ocupação são suficientes para controle de confundimento.

Nos exemplos anteriores, criou-se a hipótese de que a relação entre a variável latente (personalidade) e o estresse no trabalho é mediada pelo tipo de ocupação. Todavia, se a personalidade tem um efeito direto na exposição ao estresse no trabalho, alguns dos caminhos pela porta de trás incluirão uma variável não observada, impossi- 
Figura 5

Gráfico representando a aplicação do critério da porta de trás no diagrama da Figura $2 \mathrm{~b}$. O conjunto para ajuste contém as variáveis idade, renda e ocupação.

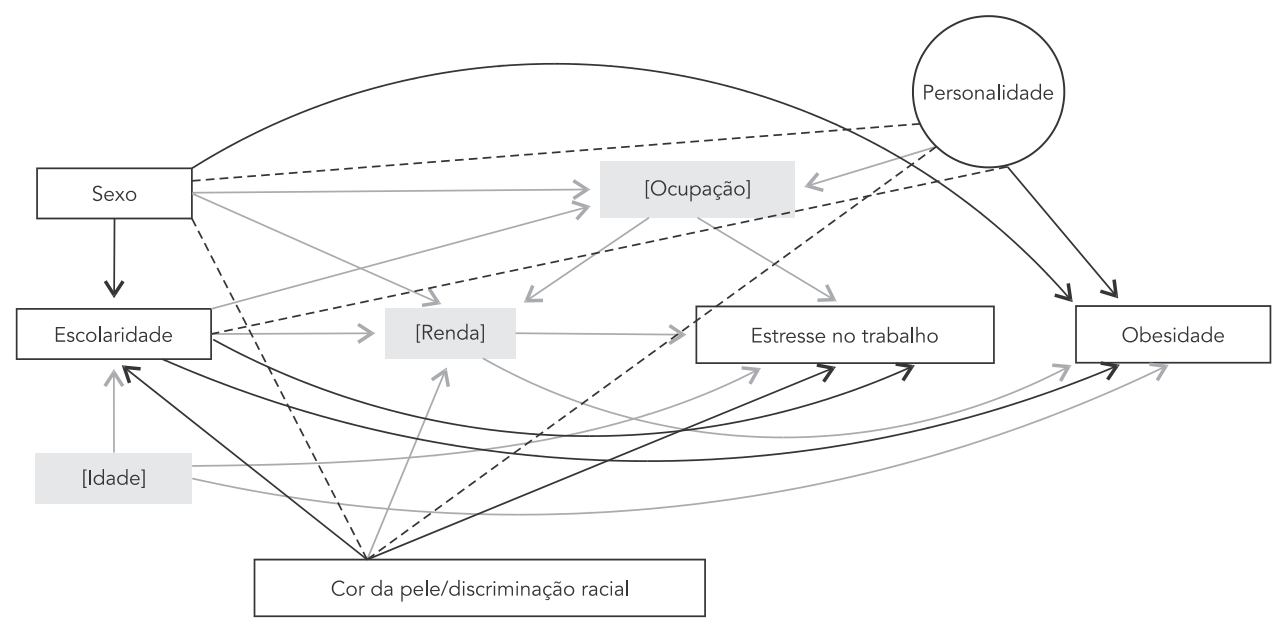

Nota: setas em negrito, destacam-se os caminhos pela porta de trás desbloqueados.

Figura 6

Gráfico representando a aplicação do critério da porta de trás no diagrama da Figura $2 \mathrm{~b}$. O conjunto para ajuste contém as variáveis idade, sexo, renda e escolaridade.

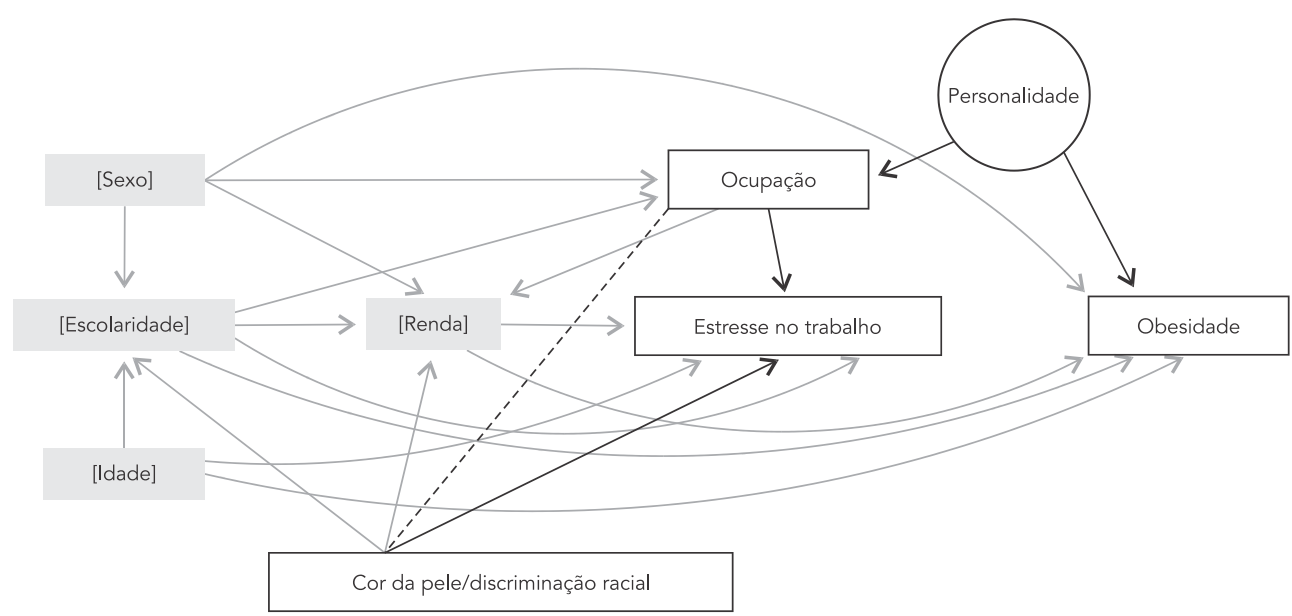

Nota: setas em negrito, destacam-se os caminhos pela porta de trás desbloqueados. 
bilitando o ajuste estatístico. Contudo, mesmo na presença de tais confundidores não mensurados, pode-se, às vezes, concluir que o efeito de uma exposição é identificável. Um método geral para identificar conjuntos suficientes para ajuste estatístico foi desenvolvido por Shpitser 17.

Outra questão importante é a necessidade de determinar se dois conjuntos candidatos para ajuste possuem o mesmo potencial de redução de viés. Para o gráfico apresentado na Figura 2a, com a aplicação do critério da porta de trás, obtêm-se dois conjuntos mínimos suficientes para o ajuste estatístico $\left(\mathrm{Sm}_{1}=\right.$ idade, renda e ocupação e $\mathrm{Sm}_{2}$ = idade, sexo, renda e escolaridade). Se a estrutura causal representada no diagrama está correta, então os dois conjuntos de covariáveis implicam a mesma redução do viés de confusão 10 . No entanto, outros aspectos como dimensionalidade, erros de mensuração e variabilidade da amostra são passíveis de influenciar a estimativa de efeito 29. Contudo, a natureza não paramétrica dos diagramas causais implica que alguns desses fatores não podem ser facilmente considerados pela teoria gráfica básica.

Com base nas formalizações teóricas apresentadas em Pearl \& Paz 29 e Greenland \& Pearl 30 , abordagens alternativas para seleção de variáveis para ajuste têm sido propostas utilizando a combinação de diagramas causais e procedimentos estatísticos tradicionais 21 .

Os diagramas causais podem ainda gerar conclusões úteis, mesmo em situações nas quais não é possível identificar um conjunto suficiente de variáveis para o controle de confundimento. Por exemplo, quando o controle para fatores de confusão é inadequado, resultados qualitativos, como a direção do viés, podem ser obtidos, em certos casos, por intermédio de diagramas causais valorados 31 . Na presença de múltiplos vieses, outras estratégias quantitativas podem ser adotadas. Greenland 32 apresenta uma revisão sobre diferentes técnicas para modelagem de viés na análise de estudos observacionais.

\section{Considerações finais}

A análise causal em estudos observacionais requer, ao menos, duas fontes de informação: dados empíricos e pressupostos causais. Nesse contexto, a principal contribuição da teoria dos diagramas causais é oferecer uma nova linguagem para a comunicação desses pressupostos e permitir caracterizar o que pode e não pode ser apreendido, dado o conjunto de premissas escolhidas.

Porém, as limitações práticas relacionadas ao que pode ser observado e ao conhecimento cien- tífico disponível impõem algumas dificuldades para a construção e a utilização desses modelos.

Algumas dessas dificuldades podem ser minimizadas mediante estratégias adotadas durante a fase de planejamento do estudo. Nesta fase, o exercício de construção de modelos causais plausíveis bem como a reflexão cuidadosa sobre hipóteses alternativas permitirão, por exemplo, identificar mais facilmente os dados necessários para responder a uma determinada pergunta de pesquisa, assim como o desenho de estudo mais apropriado. A construção desses modelos pode ser vista como um processo circular, pois, à medida que algumas hipóteses são falsificadas e novas evidências são obtidas, essas informações podem ser combinadas para construir e atualizar os modelos causais.

Além disso, a utilização desses diagramas na comunicação dos resultados permite que modelos concorrentes possam ser comparados, facilitando a identificação de possíveis explicações para resultados inconsistentes encontrados na literatura. Contudo, como estruturas causais diferentes nem sempre resultam em conclusões distintas sobre confundimento, a comparação entre diagramas causais pode facilitar a identificação de outras possíveis fontes de viés e incerteza na interpretação dos resultados do estudo. Nesse contexto, ressalta-se que a comunicação não transparente de qualquer incerteza sobre os modelos adotados pode contribuir para subestimação da incerteza e para uma falsa percepção de testabilidade do modelo, assim como pode impedir ou limitar a sua consideração pela comunidade científica.

Por fim, destaca-se que, independentemente da abordagem utilizada, a inferência causal em estudos observacionais quase sempre será um exercício desafiador, e a identificabilidade de um parâmetro causal sempre dependerá da validade do modelo considerado. 


\section{Colaboradores}

Todos os autores participaram do planejamento, da elaboração e da redação da versão final do manuscrito; T. R. Cortes teve a responsabilidade primária pelo conteúdo final.

\section{Referências}

1. Glymour MM, Greenland S. Causal diagrams. In: Rothman KJ, Greenland S, Lash TL, editors. Modern epidemiology. 3rd Ed. Philadelphia: Lippincott Williams \&Wilkins; 2008. p. 183-209.

2. Greenland S, Pearl J, Robins JM. Causal diagrams for epidemiologic research. Epidemiology 1999; 10:37-48.

3. Hernán MA, Hernandez-Diaz S, Robins JM. A structural approach to selection bias. Epidemiology $2004 ; 15: 615-25$.

4. Hernán MA, Cole SR. Invited commentary: causal diagrams and measurement bias. Am J Epidemiol 2009; 170:959-62.

5. Daniel RM, Kenward MG, Cousens SN, De Stavola BL. Using causal diagrams to guide analysis in missing data problems. Stat Methods Med Res 2012; 21:243-56.

6. Pearl J. The causal mediation formula - a guide to the assessment of pathways and mechanisms. Prev Sci 2012; 13:426-36.

7. Wright S. Correlation and causation. J Agric Res $1921 ; 20: 557-85$

8. Haavelmo T. The statistical implications of a system of simultaneous equations. Econometrica 1943; 11:1-12.

9. Pearl J. Causal diagrams for empirical research. Biometrika 1995; 82:669-88.

10. Pearl J. Causality: models, reasoning and inference. Cambridge: Cambridge University Press; 2000.

11. Spirtes P, Glymour C, Scheines R. Causation, prediction, and search. New York: Springer Verlag; 1993.

12. Aalen O, Borgan O, Gjessing HK. Survival and event history analysis: a process point of view. New York: Springer; 2008.

13. Clarke B, Leuridan B, Williamson J. Modelling mechanisms with causal cycles. Synthese 2014; 191:1651-81.
14. Gebharter A, Kaiser MI. Causal graphs and biological mechanisms. In: Kaiser MI, Scholz O, Plenge D Hüttemann A, editors. Explanation in the special sciences: the case of biology and history. New York: Springer; 2014. p. 55-85.

15. Robins JM. Data, design, and background knowledge in etiologic inference. Epidemiology 2001; 12:313-20.

16. Reichenbach H. The philosophy of space and time. New York: Dover; 1958.

17. Shpitser I. Complete identification methods for causal inference [Tese de Doutorado]. Los Angeles: University of California; 2008.

18. Ogburn EL, VanderWeele TJ. Causal diagrams for interference. Stat Sci 2014; 29:559-78.

19. Auchincloss AH, Roux AVD. A new tool for epidemiology: the usefulness of dynamic-agent models in understanding place effects on health. Am J Epidemiol 2008; 168:1-8.

20. Greenland S. Overthrowing the tyranny of null hypotheses hidden in causal diagrams. In: Dechter $\mathrm{R}$, Gerffner H, Halpern JY, editors. Heuristics, probability and causality: a tribute to Judea Pearl. London: College Publications; 2010. p. 365-82.

21. Evans D, Chaix B, Lobbedez T, Verger C, Flahaut A. Combining directed acyclic graphs and the change-in-estimate procedure as a novel approach to adjustment-variable selection in epidemiology. BMC Med Res Methodol 2012; 12:156.

22. Krieger N, Sidney S, Coakley E. Racial discrimination and skin color in the CARDIA study: implications for public health research. Coronary Artery Risk Development in Young Adults. Am J Public Health 1998; 88:1308-13.

23. Textor J, Hardt J, Knüppel S. DAGitty: a graphical tool for analyzing causal diagrams. Epidemiology $2011 ; 22: 745$. 
24. Knuppel S, Stang A. DAG program: identifying minimal sufficient adjustment sets. Epidemiology 2010; 21:159.

25. Kleinbaum DG, Kupper LL, Morgenstern H. Epidemiologic research: principles and quantitative methods. New York: Wiley; 1982.

26. Hernán MA, Hernández-Díaz S, Werler MM, Mitchell AA. Causal knowledge as a prerequisite for confounding evaluation: an application to birth defects epidemiology. Am J Epidemiol 2002; 155:176-84.

27. Shrier I, Platt RW. Reducing bias through directed acyclic graphs. BMC Med Res Methodol 2008; 8:70.

28. Greenland S. Quantifying biases in causal models: classical confounding vs collider-stratification bias. Epidemiology 2003; 14:300-6.
29. Pearl J, Paz A. Confounding equivalence in observational studies (or, when are two measurements equally valuable for effect estimation?). In: Proceedings of the 26th Conference on Uncertainty in Artificial Intelligence. Corvallis: Association for Uncertainty in Artificial Intelligence; 2010. p. 433-41.

30. Greenland S, Pearl J. Adjustments and their consequences - collapsibility analysis using graphical models. Int Stat Rev 2011; 79:401-26.

31. VanderWeele TJ, Hernán MA, Robins JM. Causal directed acyclic graphs and the direction of unmeasured confounding bias. Epidemiology 2008; 19:720-8.

32. Greenland S. Multiple bias modelling for analysis of observational data. J R Stat Soc Ser A Stat Soc 2005; 168:267-306.

\begin{abstract}
Epidemiological research still rarely uses causal diagrams, despite growing recognition of their explanatory potential. One possible reason is that many research programs involve themes in which there is a certain degree of uncertainty as to mechanisms in the processes that generate the data. In this study, the relationship between occupational stress and obesity is used as an example of the application of causal diagrams to questions related to confounding. The article presents the selection stages for variables in statistical adjustment and the derivation of a causal diagram's statistical implications. The main advantage of causal diagrams is that they explicitly reveal the respective model's underlying hypotheses, allowing critical analysis of the implications and thereby facilitating identification of sources of bias and uncertainty in the epidemiological study's results.
\end{abstract}

Causality; Epidemiologic Methods; Bias

(Epidemiology); Statistical Models

\section{Resumen}

A pesar del creciente reconocimiento de su potencial, los diagramas causales todavía se utilizan poco en la investigación epidemiológica. Una de las posibles razones es que muchos programas de investigación están involucrados en temas sobre los cuales existe un cierto grado de incertidumbre acerca de los mecanismos de los procesos que generan los datos. En este trabajo, la relación entre estrés ocupacional y obesidad se utiliza como un ejemplo de aplicación de diagramas causales en cuestiones relacionadas con la confusión. Se presentan etapas de la selección de variables para el ajuste estadístico y de la derivación de las implicaciones estadísticas de un diagrama causal. La principal ventaja de los diagramas causales es hacer explícitas las hipótesis adyacentes al modelo considerado, permitiendo que sus implicaciones puedan ser analizadas críticamente, facilitando, de esta forma, la identificación de posibles fuentes de sesgo e incertidumbre en los resultados de un estudio epidemiológico.

Causalidad; Métodos Epidemiológicos; Sesgo

(Epidemiología); Modelos Estadísticos
Recebido em 26/Jun/2015

Versão final reapresentada em 19/Fev/2016

Aprovado em 11/Mar/2016 